\title{
Malliavin Derivatives in Spaces with Variable Exponents
}

\author{
Bochi Xu, Yongqiang Fu, and Boping Tian \\ Department of Mathematics, Harbin Institute of Technology, Harbin 150001, China \\ Correspondence should be addressed to Bochi Xu; xubochi@126.com
}

Received 22 January 2014; Accepted 13 March 2014; Published 3 April 2014

Academic Editor: Donghai Ji

Copyright (C) 2014 Bochi Xu et al. This is an open access article distributed under the Creative Commons Attribution License, which permits unrestricted use, distribution, and reproduction in any medium, provided the original work is properly cited.

Spaces with variable exponents $L^{p(x)}(H, \mu)$ and $L^{p(x)}(H, \mu ; H)$ are introduced. After discussing some approximation results of $L^{p(x)}(H, \mu)$, Sobolev spaces on $H$ with variable exponents are introduced. At last, we define Malliavin derivatives in $L^{p(x)}(H, \mu)$ and discuss some properties of Malliavin derivatives in $L^{p(x)}(H, \mu)$.

\section{Introduction}

Variable exponent spaces play an important role in the study of some nonlinear problems in natural science and engineering. In decades, there is a rapid development on the subject of variable exponent function spaces. Basic properties of the spaces have been discussed by Kováčik and Rákosník in [1]. Some theories of variable exponent spaces can also be found in $[2,3]$. Harjulehto et al. present an overview of applications to differential equations with nonstandard growth in [4]. Diening et al. [5] summarize most of the existing literature of theory of function spaces with variable exponents and applications to partial differential equations. In [6], Aoyama discusses the properties of Lebesgue spaces with variable exponents on a probability space.

Malliavin derivatives have many applications on mathematical finance (see Malliavin and Thalmaier [7] and di Nunno et al. [8]), and we are interested in the behavior of Malliavin derivatives in spaces with variable exponents. In this paper, in Section 2, motivated by $[6,9,10]$, we will first introduce $L^{p(x)}(H, \mu)$ and $L^{p(x)}(H, \mu ; H)$ and give some approximation results of $L^{p(x)}(H, \mu)$, which are useful in the definition on Malliavin derivatives in the following parts. In Section 3 we discuss gradients in $L^{p(x)}(H, \mu)$ and give properties of the linear operator $D$. At the end of this section, we define variable exponent Sobolev spaces on $H$. After the above preparation, we give Malliavin derivatives in $L^{p(x)}(H, \mu)$ and discuss some properties of the Malliavin derivative operator $M$ in $L^{p(x)}(H, \mu)$ in the last section.

\section{Preliminaries}

Let $H$ be a separable Hilbert space. A Borel probability measure $N_{m, Q}$ in $H$ with mean $m$ and compact covariance operator $Q$ is called Gaussian measure if the Fourier transform satisfies

$$
\int_{H} e^{i\langle h, x\rangle} N_{m, Q}(d x)=e^{i\langle m, h\rangle} e^{(-1 / 2)\langle Q h, h\rangle}, \quad \forall x \in H .
$$

$N_{m, Q}$ is called nondegenerate if KerQ $=\{0\}$. We are given a nondegenerate Gaussian measure $\mu=N_{0, Q}$ in the Hilbert space $H$. Since the operator $Q$ is compact, there exists a complete orthonormal system $\left\{e_{k}\right\}$ in $H$ and a sequence $\left\{\lambda_{k}\right\}$ of positive numbers such that

$$
Q e_{k}=\lambda_{k} e_{k}, \quad k \in \mathbb{N} .
$$

We denote by $C_{b}(H)$ the space of all mappings $\phi: H \rightarrow \mathbb{R}$, which are both continuous and bounded. $C_{b}(H)$ is a Banach space with the norm $\|\varphi\|_{0}=\sup _{x \in H}|\varphi(x)|$. And denote by $C_{b}^{k}(H), k \in \mathbb{N}$, the space of all mappings $\phi: H \rightarrow \mathbb{R}$ which are continuous and bounded together with their derivatives of order not bigger than $k$.

Given a variable exponent $p: H \rightarrow[1, \infty]$, it is assumed to be a Borel measurable function. On the set of all 
Borel measurable functions, the moduli $\rho$ and $\tilde{\rho}$ are defined, respectively, by

$$
\begin{aligned}
& \rho(\varphi)=\int_{H \backslash H_{\infty}}|\varphi(x)|^{p(x)} \mu(d x)+\text { ess } \sup _{x \in H_{\infty}}|\varphi(x)|, \\
& \tilde{\rho}(F)=\int_{H \backslash H_{\infty}}|F(x)|_{H}^{p(x)} \mu(d x)+\text { ess } \sup _{x \in H_{\infty}}|F(x)|_{H},
\end{aligned}
$$

where $H_{\infty}=\{x: p(x)=\infty\}, \varphi: H \rightarrow \mathbb{R}$, and $F: H \rightarrow H$.

Definition 1. The space $\mathscr{L}^{p(x)}(H, \mu)$ is the set of Borel measurable functions $\varphi: H \rightarrow \mathbb{R}$ such that $\rho(\varphi)<\infty$, and it is endowed with the Luxemburg norm:

$$
\|\varphi\|_{L^{p(x)}(H, \mu)}=\inf \left\{\lambda>0: \rho\left(\frac{\varphi}{\lambda}\right) \leq 1\right\} .
$$

Define the equivalence relation $r_{1}: \varphi \sim \psi$ if and only if $\|\varphi-\psi\|_{L^{p(x)}(H, \mu)}=0$. Denote by $L^{p(x)}(H, \mu)$ the quotient $\mathscr{L}^{p(x)}(H, \mu)$ with respect to the equivalence relation $r_{1}$.

Definition 2. The space $\mathscr{L}^{p(x)}(H, \mu ; H)$ is the set of Borel measurable functions $F: H \rightarrow H$ such that $\widetilde{\rho}(F)<\infty$, and it is endowed with the Luxemburg norm:

$$
\|F\|_{L^{p(x)}(H, \mu ; H)}=\inf \left\{\lambda>0: \bar{\rho}\left(\frac{F}{\lambda}\right) \leq 1\right\} .
$$

Define the equivalence relation $r_{2}: F \sim G$ if and only if $\|F-G\|_{L^{p(x)}(H, \mu ; H)}=0$. Denote by $L^{p(x)}(H, \mu ; H)$ the quotient $\mathscr{L}^{p(x)}(H, \mu ; H)$ with respect to the equivalence relation $r_{2}$.

Proposition 3 (see Lemma 3.2.20 in [5]). Let variable exponents $p(x), q(x), r(x) \in[1, \infty]$ be such that $1 / r(x)=$ $(1 / p(x))+(1 / q(x)), \mu$-a.e.; then the inequality

$$
\|\varphi \psi\|_{L^{r(x)}(H, \mu)} \leq 2\|\varphi\|_{L^{p(x)}(H, \mu)}\|\psi\|_{L^{q(x)}(H, \mu)}
$$

holds for every $\varphi \in L^{p(x)}(H, \mu)$ and $\varphi \in L^{q(x)}(H, \mu)$, where in the case $r=p=q=\infty$ we use the convention $r / p=r / q=1$.

Proposition 4. The variable exponent $p$ satisfies $1 \leq p^{-} \leq$ $p(x) \leq p^{+}<\infty$, $\mu$-a.e. Let $F \in L^{p(x)}(H, \mu ; H)$ and set $F_{k}(x)=$ $\left\langle F(x), e_{k}\right\rangle ; k \in \mathbb{N}$. Then $F_{k} \in L^{p(x)}(H, \mu)$ for all $k \in \mathbb{N}$ and

$$
F(x)=\sum_{k=1}^{\infty} F_{k}(x) e_{k}, \quad \mu \text {-a.e., }
$$

where the series is convergent in $L^{p(x)}(H, \mu ; H)$.

Proof. For $F_{k}, k \in \mathbb{N}$,

$$
\int_{H}\left|F_{k}(x)\right|^{p(x)} \mu(d x) \leq \int_{H}|F(x)|_{H}^{p(x)} \mu(d x)<\infty,
$$

so $F_{k} \in L^{p(x)}(H, \mu)$. Since $\left\{e_{k}\right\}$ is a complete orthonormal system in $H$ and $F(x) \in H$ for $x \in H$, we have

$$
F(x)=\sum_{k=1}^{\infty} F_{k}(x) e_{k}, \quad \mu \text {-a.e. }
$$

Set $P_{n} F(x)=\sum_{k=1}^{n} F_{k}(x) e_{k}$, and we have

$$
\begin{gathered}
\lim _{n \rightarrow \infty} P_{n} F(x)=F(x), \quad \mu \text {-a.e., } \\
|F(x)|_{H}^{p(x)}=\left(\sum_{k=1}^{\infty}\left|F_{k}(x)\right|^{2}\right)^{p(x) / 2} \geq\left(\sum_{k=n+1}^{\infty}\left|F_{k}(x)\right|^{2}\right)^{p(x) / 2} \\
=\left|P_{n} F(x)-F(x)\right|_{H}^{p(x)}
\end{gathered}
$$

so by Lebesgue's Dominated Convergence Theorem, we have

$$
\lim _{n \rightarrow \infty} \int_{H}\left|P_{n} F(x)-F(x)\right|_{H}^{p(x)} \mu(d x)=0 .
$$

Thus, the series are convergent in $L^{p(x)}(H, \mu ; H)$. The proof is completed.

Denote the linear span of all real and imaginary parts functions $\varphi_{h}(x)=e^{i\langle h, x\rangle}, x \in H$, and $h \in H$, by $\mathscr{E}(H)$.

Proposition 5 (see Lemma 2.2 in [9]). For all $\varphi \in C_{b}(H)$ there exists a two-index sequence $\left\{\varphi_{n, k}\right\} \subset \mathscr{E}(H)$ such that $\left\|\varphi_{n, k}\right\|_{0} \leq$ $\|\varphi\|_{0}, n, k \in \mathbb{N}$, and

$$
\lim _{n \rightarrow \infty} \lim _{k \rightarrow \infty} \varphi_{n, k}(x)=\varphi(x), \quad x \in H .
$$

$C_{c}(H)$ is the space of all mappings $\varphi: H \rightarrow \mathbb{R}$ which are continuous and have compact support in $H$. We have the following proposition about $C_{c}(H)$.

Proposition 6. If the variable exponent $p$ satisfies $1 \leq p^{-} \leq$ $p(x) \leq p^{+}<\infty$, $\mu$-a.e., then $C_{c}(H)$ is dense in $L^{p(x)}(H, \mu)$.

Proof. Note that $S:=S(H, \mu)$ is the set of all the simple functions. It is easy to check that $S \subset L^{p(x)}(H, \mu)$. Let $f \in L^{p(x)}(H, \mu)$ with $f \geq 0$. Since $f$ is a Borel measurable function, there exist $\left\{f_{n}\right\} \subset S$ with $0 \leq f_{n} \nearrow f$, $\mu$-a.e., and $\left|f_{n}(x)-f(x)\right|^{p(x)} \leq|f(x)|^{p(x)}$, so by Lebesgue's Dominated Convergence Theorem, we have $f_{n} \rightarrow f$ in $L^{p(x)}(H, \mu)$. Thus, $f$ is in the closure of $S$. If we drop the assumption $f \geq 0$, then we split $f$ into positive and negative parts which belong to the closure of $S$. Thus $S$ is dense in $L^{p(x)}(H, \mu)$. For any $\varepsilon>0$ and $g \in L^{p(x)}(H, \mu)$, there exists $s \in S$ such that $\|g-s\|_{L^{p(x)}(H, \mu)}<\varepsilon / 2$.

On the other hand, for $p^{+}<\infty, C_{c}(H)$ is dense in $L^{p^{+}+1}(H, \mu)$. So there exists $\widetilde{g} \in C_{c}(H)$ such that $\|s-\tilde{g}\|_{L^{p^{+}+1}(H, \mu)}<\varepsilon / 4$. By Proposition 3, we have

$$
\|s-\tilde{g}\|_{L^{p(x)}(H, \mu)} \leq 2\|s-\tilde{g}\|_{L^{p^{+}+1}(H, \mu)}<\frac{\varepsilon}{2} .
$$

Thus, $\|g-\tilde{g}\|_{L^{p(x)}(H, \mu)} \leq \varepsilon$ and $C_{c}(H)$ is dense in $L^{p(x)}(H, \mu)$. The proof is completed.

Proposition 7. If the variable exponent $p$ satisfies $1 \leq p^{-} \leq$ $p(x) \leq p^{+}<\infty, \mu$-a.e., then $\mathscr{E}(H)$ is dense in $L^{p(x)}(H, \mu)$. 
Proof. Since $C_{c}(H) \subset C_{b}(H)$, by Proposition $6 C_{b}(H)$ is dense in $L^{p(x)}(H, \mu)$. So for any $\varepsilon>0$ and $f \in L^{p(x)}(H, \mu)$, there exists $\varphi \in C_{b}(H)$ such that

$$
\|f-\varphi\|_{L^{p(x)}(H, \mu)}<\frac{\varepsilon}{2} .
$$

By Proposition 5 and diagonal rule, there exists a sequence $\left\{\varphi_{n}\right\} \subset \mathscr{E}(H)$ such that $\left|\varphi_{n}(x)\right| \leq\|\varphi\|_{0}, n \in \mathbb{N}$, and

$$
\lim _{n \rightarrow \infty} \varphi_{n}(x)=\varphi(x), \quad x \in H .
$$

By Lebesgue's Dominated Convergence Theorem, we have

$$
\lim _{n \rightarrow \infty} \int_{H}\left|\varphi_{n}(x)-\varphi(x)\right| \mu(d x)=0 .
$$

And as

$$
\begin{aligned}
& \int_{H}\left|\varphi_{n}(x)-\varphi(x)\right|^{p(x)} \mu(d x) \\
& \quad \leq\left(2\|\varphi\|_{0}\right)^{p^{+}} \int_{H}\left|\varphi_{n}(x)-\varphi(x)\right| \mu(d x),
\end{aligned}
$$

we have $\varphi_{n} \rightarrow \varphi$ in $L^{p(x)}(H, \mu)$. Suppose that $N \in$ $\mathbb{N},\left\|\varphi_{n}-\varphi\right\|_{L^{p(x)}(H, \mu)}<\varepsilon / 2$ for $n>N$, and we have $\left\|\varphi_{n}-f\right\|_{L^{p(x)}(H, \mu)}<\varepsilon$ for $n>N$. Thus, $\mathscr{E}(H)$ is dense in $L^{p(x)}(H, \mu)$.

Proposition 8. Suppose that $W_{z}, z \in H$, is a white noise function on $H$. If the variable exponent $p$ satisfies $1 \leq p^{-} \leq$ $p(x) \leq p^{+}<\infty, \mu$-a.e., then

$$
\left\|W_{z}\right\|_{L^{p(x)}(H, \mu)} \leq 2\left(\frac{(2 m) !}{2^{m} m !}|z|_{H}^{m}\right)^{1 / 2 m}
$$

where $p^{+}<2 m$ and $m \in \mathbb{N}$.

Proof. Since $W_{z}$ is a Gaussian random variable with mean 0 and covariance $|z|_{H}$, by Proposition 3, we have

$$
\begin{aligned}
\left\|W_{z}\right\|_{L^{p(x)}(H, \mu)} & \leq 2\|1\|_{L^{(2 m p(x)) /(2 m-p(x))}(H, \mu)}\left\|W_{z}\right\|_{L^{2 m}(H, \mu)} \\
& =2\left(\frac{(2 m) !}{2^{m} m !}|z|_{H}^{m}\right)^{1 / 2 m} .
\end{aligned}
$$

The proof is completed.

\section{Variable Exponent Sobolev Spaces on Separable Hilbert Space $H$}

For any $\varphi \in \mathscr{E}(H)$ and $k \in \mathbb{N}$, we denote by $D_{k} \varphi$ its derivative in the direction of $e_{k}$; that is,

$$
D_{k} \varphi(x)=\lim _{\varepsilon \rightarrow 0} \frac{1}{\varepsilon}\left(\varphi\left(x+\varepsilon e_{k}\right)-\varphi(x)\right), \quad x \in H .
$$

Denote the gradient of $\varphi$ by $D \varphi$.

We will consider the following linear mapping:

$$
D: \mathscr{E}(H) \subset L^{p(x)}(H, \mu) \longrightarrow L^{p(x)}(H, \mu ; H), \quad \varphi \longmapsto D \varphi,
$$

Lemma 9 (see Lemma 2.6 in [9]). Suppose $\varphi, \psi \in \mathscr{E}(H)$; then

$$
\int_{H} D_{k} \varphi \psi d \mu=-\int_{H} \varphi D_{k} \psi d \mu+\frac{1}{\lambda_{k}} \int_{H} x_{k} \varphi \psi d \mu,
$$

where $x \in H$ and $x_{k}=\left\langle x, e_{k}\right\rangle$.

Lemma 10 (see Corollary 2.7 in [9]). Suppose $\varphi, \psi \in \mathscr{E}(H)$ and $z \in Q^{1 / 2}(H)$; then

$$
\int_{H}\langle D \varphi, z\rangle \psi d \mu=-\int_{H}\langle D \psi, z\rangle \varphi d \mu+\int_{H} W_{\mathrm{Q}^{-1 / 2} z} \varphi \psi d \mu .
$$

Given a linear operator $A$, not necessarily closed, if the closure of its graph happens to be the graph of some operator, that operator is called the closure of $A$, and we say that $A$ is closable. Denote the closure of $A$ by $\bar{A}$. And $A$ is closable if and only if for any sequence $\left\{x_{n}\right\} \subset D(A)$ such that

$$
\lim _{n \rightarrow \infty} x_{n}=0, \quad \lim _{n \rightarrow \infty} A x_{n}=y,
$$

one has $y=0$.

Theorem 11. The mapping $D$ is a closable linear operator.

Proof. Let $\left\{\varphi_{n}\right\} \subset \mathscr{E}(H)$ such that

$$
\begin{aligned}
& \varphi_{n} \longrightarrow 0 \quad \text { in } L^{p(x)}(H, \mu), \\
& D \varphi_{n} \longrightarrow F \quad \text { in } L^{p(x)}(H, \mu ; H) .
\end{aligned}
$$

By the definition of closable operators, we only need to prove that $F=0$.

For any $\psi \in \mathscr{E}(H)$ and $z \in Q^{1 / 2}(H)$, by Lemma 10, we have

$$
\int_{H}\left\langle D \varphi_{n}, z\right\rangle \psi d \mu=-\int_{H}\langle D \psi, z\rangle \varphi_{n} d \mu+\int_{H} W_{\mathrm{Q}^{-1 / 2} z} \varphi_{n} \psi d \mu .
$$

By Proposition 3, we have

$$
\begin{gathered}
\left|\int_{H}\left\langle D \varphi_{n}-F, z\right\rangle \psi d \mu\right| \leq \int_{H}\left|D \varphi_{n}-F\right|_{H}|z|_{H}|\psi|_{H} d \mu \\
\leq 2|z|_{H}\left\|D \varphi_{n}-F\right\|_{L^{p(x)}(H, \mu ; H)} \\
\times\|\psi\|_{L^{q(x)}(H, \mu)}, \\
\left|\int_{H}\langle D \psi, z\rangle \varphi_{n} d \mu\right| \leq 2|z|_{H}\left\|\varphi_{n}\right\|_{L^{p(x)}(H, \mu)}\|D \psi\|_{L^{q(x)}(H, \mu ; H)},
\end{gathered}
$$

where $(1 / p(x))+(1 / q(x))=1 ; x \in H$. Since $\psi$ is bounded, by Propositions 3 and 8 , we have

$$
\left|\int_{H} W_{Q^{-1 / 2} z} \varphi_{n} \psi d \mu\right| \leq 2 C\left\|\varphi_{n}\right\|_{L^{p(x)}(H, \mu)}\left\|W_{Q^{-1 / 2} z}\right\|_{L^{q(x)}(H, \mu)}
$$

$$
\leq C_{p^{+}}\left|Q^{-1 / 2} z\right|_{H}^{1 / 2}\left\|\varphi_{n}\right\|_{L^{p(x)}(H, \mu)} .
$$

where $1 \leq p^{-} \leq p(x) \leq p^{+}<\infty, \mu$-a.e. 
Thus, $\int_{H}\langle F, z\rangle \psi d \mu=0$, as $n \rightarrow \infty$. Since $F_{k} \in L^{p(x)}(H, \mu)$, for a fixed $k \in \mathbb{N}$, suppose $\left\{\psi_{k n}\right\} \subset \mathscr{E}(H)$ such that $\psi_{k n} \rightarrow$ $\left|F_{k}\right|^{p(x)-1} \operatorname{sgn}\left(F_{k}\right)$ in $L^{q(x)}(H, \mu)$. We have

$$
\lim _{n \rightarrow \infty} \int_{H} F_{k} \psi_{k n} d \mu=0
$$

that is, $\int_{H}\left|F_{k}\right|^{p(x)} d \mu=0$. Thus, we have $F_{k}(x)=0, \mu$-a.e., and $F(x)=0, \mu$-a.e. The proof is completed.

We denote by $\bar{D}$ the closable operator of $D$ and by $W^{1, p(x)}(H, \mu)$ the domain of $\bar{D}$.

Proposition 12. For any $k \in \mathbb{N}$, the linear operator $D_{k}$ is closable and its closure $\bar{D}_{k}$ satisfies $\bar{D}_{k} \varphi=\left\langle\bar{D} \varphi, e_{k}\right\rangle$ for $\varphi \epsilon$ $W^{1, p(x)}(H, \mu)$.

Proof. For any $k \in \mathbb{N}$, let $\left\{\varphi_{n}\right\} \subset \mathscr{E}(H)$ such that

$$
\begin{aligned}
& \varphi_{n} \longrightarrow 0 \text { in } L^{p(x)}(H, \mu), \\
& D_{k} \varphi_{n} \longrightarrow F_{k} \text { in } L^{p(x)}(H, \mu) .
\end{aligned}
$$

By the definition of closable operators, we only need to prove that $F_{k}=0$. For any $\psi \in \mathscr{E}(H)$ and $z \in H$, by Lemma 9 , we have

$$
\int_{H} D_{k} \varphi_{n} \psi d \mu=-\int_{H} \varphi_{n} D_{k} \psi d \mu+\frac{1}{\lambda_{k}} \int_{H} x_{k} \varphi_{n} \psi d \mu .
$$

Similar to the proof of Theorem 11, we have $\int_{H} F_{k} \psi d \mu=0$ as $n \rightarrow \infty$ and $F_{k}=0$, $\mu$-a.e. Thus $D_{k}$ is closable. And

$$
\bar{D}_{k} \varphi=\lim _{n \rightarrow \infty} D_{k} \varphi_{n}=\lim _{n \rightarrow \infty}\left\langle D \varphi_{n}, e_{k}\right\rangle=\left\langle\bar{D} \varphi, e_{k}\right\rangle .
$$

The proof is completed.

\section{Malliavin Derivatives in $D^{1, p(x)}(H, \mu)$}

For any $\varphi \in \mathscr{E}(H)$, define the linear operator:

$$
\begin{aligned}
& M_{0}: \mathscr{E}(H) \subset L^{p(x)}(H, \mu) \longrightarrow L^{p(x)}(H, \mu ; H), \\
& M_{0} \varphi(x)=Q^{1 / 2} D \varphi(x), \quad \varphi \in \mathscr{E}(H), x \in H,
\end{aligned}
$$

where $1 \leq p^{-} \leq p(x) \leq p^{+}<\infty, \mu$-a.e. And

$$
M_{k} \varphi(x)=\left\langle M_{0} \varphi(x), e_{k}\right\rangle=\lambda_{k}^{1 / 2} D_{k} \varphi(x),
$$

where $\varphi \in \mathscr{E}(H)$ and $x \in H$.

Proposition 13 (see Corollary 2.10 in [9]). Suppose $\varphi, \psi \in$ $\mathscr{E}(H)$ and $z \in H$; then the following identity holds:

$$
\int_{H}\left\langle M_{0} \varphi, z\right\rangle \psi d \mu=-\int_{H}\left\langle M_{0} \psi, z\right\rangle \varphi d \mu+\int_{H} W_{z} \varphi \psi d \mu .
$$

In a similar way to Theorem 11, one has Theorem 14.

Theorem 14. The mapping $M_{0}$ is a closable linear operator.
Proof. Let $\left\{\varphi_{n}\right\} \subset \mathscr{E}(H)$ such that

$$
\begin{aligned}
& \varphi_{n} \longrightarrow 0 \text { in } L^{p(x)}(H, \mu), \\
& M_{0} \varphi_{n} \longrightarrow F \text { in } L^{p(x)}(H, \mu ; H) .
\end{aligned}
$$

We only need to prove that $F=0$.

For any $\psi \in \mathscr{E}(H)$ and $z \in H$, by Proposition 13, we have

$$
\int_{H}\left\langle M_{0} \varphi_{n}, z\right\rangle \psi d \mu=-\int_{H}\left\langle M_{0} \psi, z\right\rangle \varphi_{n} d \mu+\int_{H} W_{z} \varphi_{n} \psi d \mu .
$$

And we have $\int_{H}\langle F, z\rangle \psi d \mu=0$, as $n \rightarrow \infty$. Similar to Theorem 11, we have $F(x)=0$, $\mu$-a.e. The proof is completed.

We denote by $M$ the closable operator of $M_{0}$ and by $D^{1, p(x)}(H, \mu)$ the domain of $M$. For any $\varphi \in D^{1, p(x)}(H, \mu)$, we call $M \varphi$ the Malliavin derivative of $\varphi$.

Proposition 15. If $p$ satisfies $1 \leq p(x) \leq p^{+}<\infty$, $\mu$-a.e., then $W^{1, p(x)}(H, \mu) \subset D^{1, p(x)}(H, \mu)$ and $M \varphi=Q^{1 / 2} \bar{D} \varphi$ for any $\varphi \in W^{1, p(x)}(H, \mu)$.

Proof. For any $\varphi \in W^{1, p(x)}(H, \mu)$, there exists $\left\{\varphi_{n}\right\} \subset \mathscr{E}(H)$ such that $\varphi_{n} \rightarrow \varphi$ in $L^{p(x)}(H, \mu)$. As

$$
\begin{aligned}
& \int_{H}\left|Q^{1 / 2} D \varphi_{n}(x)-Q^{1 / 2} \bar{D} \varphi(x)\right|_{H}^{p(x)} \mu(d x) \\
& \leq \int_{H}\left\|Q^{1 / 2}\right\|^{p(x)}\left|D \varphi_{n}(x)-\bar{D} \varphi(x)\right|_{H}^{p(x)} \mu(d x) \\
& \leq \max \left\{\left\|Q^{1 / 2}\right\|,\left\|Q^{1 / 2}\right\|^{p^{+}}\right\} \int_{H}\left|D \varphi_{n}(x)-\bar{D} \varphi(x)\right|_{H}^{p(x)} \mu(d x)
\end{aligned}
$$

we have $M \varphi=Q^{1 / 2} \bar{D} \varphi$ and $\varphi \in D^{1, p(x)}(H, \mu)$.

To prove Proposition 17, we need the following lemma.

Lemma 16 (see Lemma 2.3 in [9]). For all $\varphi \in C_{b}^{1}(H)$ there exists a two-index sequence $\left\{\varphi_{n, k}\right\} \subset \mathscr{E}(H)$ such that $\left\|\varphi_{n, k}\right\|_{0}+$ $\left\|D \varphi_{n, k}\right\|_{0} \leq\|\varphi\|_{0}+\|D \varphi\|_{0}, n, k \in \mathbb{N}$, and

$$
\begin{gathered}
\lim _{n \rightarrow \infty} \lim _{k \rightarrow \infty} \varphi_{n, k}(x)=\varphi(x), \quad x \in H, \\
\lim _{n \rightarrow \infty} \lim _{k \rightarrow \infty} D \varphi_{n, k}(x)=D \varphi(x), \quad x \in H .
\end{gathered}
$$

Proposition 17. If $p$ satisfies $1 \leq p(x) \leq p^{+}<\infty$, $\mu$-a.e., then one has $g(\varphi) \in D^{1, p(x)}(H, \mu)$ and $M g(\varphi)=g^{\prime}(\varphi) M \varphi$ for any $\varphi \in D^{1, p(x)}(H, \mu)$ and $g \in C_{b}^{1}(H)$.

Proof. First, we prove that $g(\psi) \in D^{1, p(x)}(H, \mu)$ for any $\psi \in \mathscr{E}(H)$. By Lemma 16, for $g(\psi)$, there exists a two-index sequence $\left\{\phi_{n, k}\right\} \subset \mathscr{E}(H)$ such that $\left\|\phi_{n, k}\right\|_{0}+\left\|D \phi_{n, k}\right\|_{0} \leq$ $\|g(\psi)\|_{0}+\|D g(\psi)\|_{0}, n, k \in \mathbb{N}$, and

$$
\begin{gathered}
\lim _{n \rightarrow \infty} \lim _{k \rightarrow \infty} \phi_{n, k}(x)=g(\psi)(x), \quad x \in H, \\
\lim _{n \rightarrow \infty} \lim _{k \rightarrow \infty} D \phi_{n, k}(x)=D g(\psi)(x), \quad x \in H .
\end{gathered}
$$


By diagonal rule and Lebesgue's Dominated Convergence Theorem, $\phi_{n} \rightarrow g(\psi)$ in $L^{p(x)}(H, \mu)$. Since $M$ is closed, we have $M g(\psi)=\lim _{n \rightarrow \infty} M_{0} \phi_{n}$ and $g(\psi) \in D^{1, p(x)}(H, \mu)$.

Now we will prove the proposition. For $\varphi \in D^{1, p(x)}(H, \mu)$, there exists $\left\{\varphi_{n}\right\} \subset \mathscr{E}(H)$ such that

$$
\lim _{n \rightarrow \infty} \varphi_{n}=\varphi \text { in } L^{p(x)}(H, \mu) .
$$

By the first part of this proof, $g\left(\varphi_{n}\right) \in D^{1, p(x)}(H, \mu)$. And

$$
M g\left(\varphi_{n}\right)=Q^{1 / 2} D g\left(\varphi_{n}\right)=g^{\prime}\left(\varphi_{n}\right) Q^{1 / 2} D \varphi_{n}=g^{\prime}\left(\varphi_{n}\right) M \varphi_{n} .
$$

Since $M \varphi_{n} \in L^{p(x)}(H, \mu ; H)$ and $g^{\prime}\left(\varphi_{n}\right) \in C_{b}(H)$, we have

$$
\lim _{n \rightarrow \infty} M g\left(\varphi_{n}\right)=\lim _{n \rightarrow \infty} g^{\prime}\left(\varphi_{n}\right) M \varphi_{n}=g^{\prime}(\varphi) M \varphi .
$$

As $M$ is closed, $M g(\varphi)=g^{\prime}(\varphi) M \varphi$. The proof is completed.

Proposition 18. Assume that $p$ satisfies $1 \leq p(x) \leq p^{+}<$ $\infty$, $\mu$-a.e. For $\varphi, \psi \in D^{1, p(x)}(H, \mu)$; suppose $\psi$ and $M \psi$ are bounded; then $\varphi \psi \in D^{1, p(x)}(H, \mu)$ and $M(\varphi \psi)=\psi M \varphi+$ $\varphi M \psi$.

Proof. First, for $\varphi \in \mathscr{E}(H)$, as $\psi \in D^{1, p(x)}(H, \mu)$, there exists $\left\{\psi_{n}\right\} \subset \mathscr{E}(H)$ such that $\psi_{n} \rightarrow \psi$ in $L^{p(x)}(H, \mu)$. And since

$$
M_{0}\left(\varphi \psi_{n}\right)=\psi_{n} M_{0} \varphi+\varphi M_{0} \psi_{n}
$$

we have

$$
\lim _{n \rightarrow \infty} M_{0}\left(\varphi \psi_{n}\right)=\lim _{n \rightarrow \infty}\left(\psi_{n} M_{0} \varphi+\varphi M_{0} \psi_{n}\right)=\psi M_{0} \varphi+\varphi M \psi .
$$

As $\varphi \psi_{n} \in \mathscr{E}(H), \varphi \psi_{n} \rightarrow \varphi \psi$ in $L^{p(x)}(H, \mu)$, and $M(\varphi \psi)=\lim _{n \rightarrow \infty} M_{0}\left(\varphi \psi_{n}\right)$ in $L^{p(x)}(H, \mu ; H)$, we have $\varphi \psi \epsilon$ $D^{1, p(x)}(H, \mu)$.

Secondly, for $\varphi \in D^{1, p(x)}(H, \mu)$, there exists $\left\{\varphi_{n}\right\} \subset \mathscr{E}(H)$ such that $\varphi_{n} \rightarrow \varphi$ in $L^{p(x)}(H, \mu)$. By the first part of the proof,

$$
M\left(\varphi_{n} \psi\right)=\psi M_{0} \varphi_{n}+\varphi_{n} M \psi .
$$

Since $\psi$ and $M \psi$ are bounded, we have

$$
M(\varphi \psi)=\lim _{n \rightarrow \infty} M_{0}\left(\varphi_{n} \psi\right)=\psi M \varphi+\varphi M \psi,
$$

and $\varphi \psi \in D^{1, p(x)}(H, \mu)$. The proof is completed.

\section{Conclusion}

In this work some new results on Malliavin derivatives are given. Malliavin derivatives are extended from $D^{1,2}(H, \mu)$ to variable exponent spaces and some properties of Malliavin derivatives in variable exponent spaces are obtained. We introduce $L^{p(x)}(H, \mu)$ and $L^{p(x)}(H, \mu ; H)$, and this work generalizes classical variable exponents spaces based on Kováčik and Rákosník [1]. Then, we prove that $\mathscr{E}(H)$ is dense in $L^{p(x)}(H, \mu)$ and by this approximation result we give extension of Malliavin derivatives which are given by P. Malliavin [11]. Some properties of Malliavin derivatives in $D^{1,2}(H, \mu)$ are also discussed.

\section{Conflict of Interests}

The authors declare that there is no conflict of interests regarding the publication of this paper.

\section{Acknowledgment}

This work was supported by the National Natural Science Foundation of China (Grant no. 11371110).

\section{References}

[1] O. Kováčik and J. Rákosník, "On spaces $L^{p(x)}$ and $W^{k, p(x), "}$ Czechoslovak Mathematical Journal, vol. 41, no. 4, pp. 592-618, 1991.

[2] L. Diening, "Maximal function on generalized Lebesgue spaces $L^{p(\cdot), " ~ M a t h e m a t i c a l ~ I n e q u a l i t i e s ~ \& ~ A p p l i c a t i o n s, ~ v o l . ~ 7, ~ n o . ~ 2, ~ p p . ~}$ 245-253, 2004.

[3] T. Futamura, Y. Mizuta, and T. Shimomura, "Sobolev embeddings for variable exponent Riesz potentials on metric spaces," Annales Academioe Scientiarum Fennicoe. Mathematica, vol. 31, no. 2, pp. 495-522, 2006.

[4] P. Harjulehto, P. Hästö, U. V. Le, and M. Nuortio, "Overview of differential equations with non-standard growth," Nonlinear Analysis: Theory, Methods \& Applications, vol. 72, no. 12, pp. 4551-4574, 2010.

[5] L. Diening, P. Harjulehto, P. Hästö, and M. Rüžička, Lebesgue and Sobolev Spaces with Variable Exponents, Springer, 2010.

[6] H. Aoyama, "Lebesgue spaces with variable exponent on a probability space," Hiroshima Mathematical Journal, vol. 39, no. 2, pp. 207-216, 2009.

[7] P. Malliavin and A. Thalmaier, Stochastic Calculus of Variations in Mathematical Finance, World Publishing Company, Springer, 2007.

[8] G. di Nunno, B. Øksendal, and F. Proske, Malliavin Calculus for Lévy Processes with Applications to Finance, Universitext, Springer, 2009.

[9] G. da Prato, Introduction to Stochastic Analysis and Malliavin Calculus, vol. 6, Scuola Normale Superiore, Pisa, Italy, 2007.

[10] Y. Q. Fu, "Weak solution for obstacle problem with variable growth," Nonlinear Analysis: Theory, Methods \& Applications, vol. 59, no. 3, pp. 371-383, 2004.

[11] P. Malliavin, "Stochastic calculus of variations and hypoelliptic operators," in Proceedings of the International Symposium on Stochastic Differential Equations, pp. 193-263, Kyoto, Japan, 1976. 


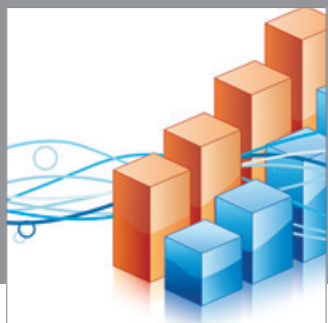

Advances in

Operations Research

mansans

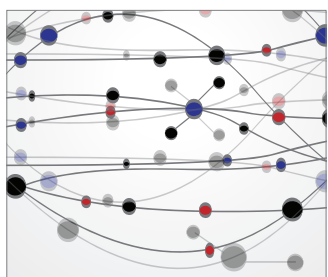

The Scientific World Journal
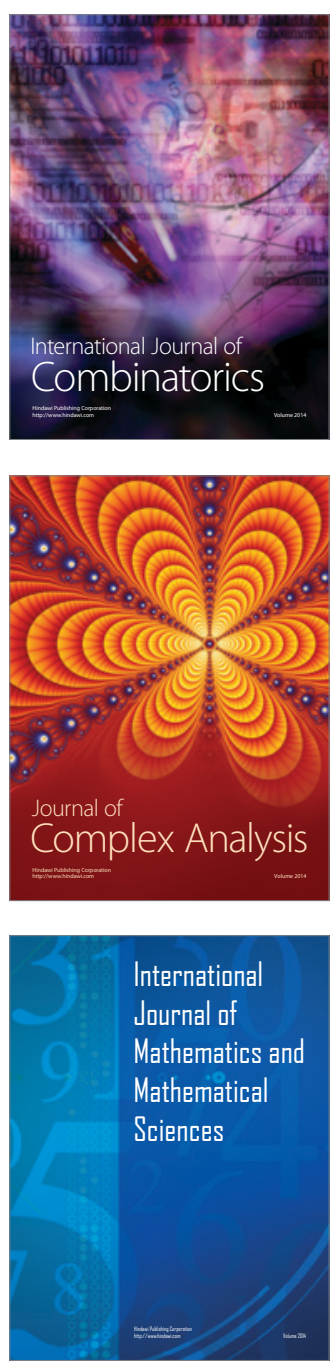
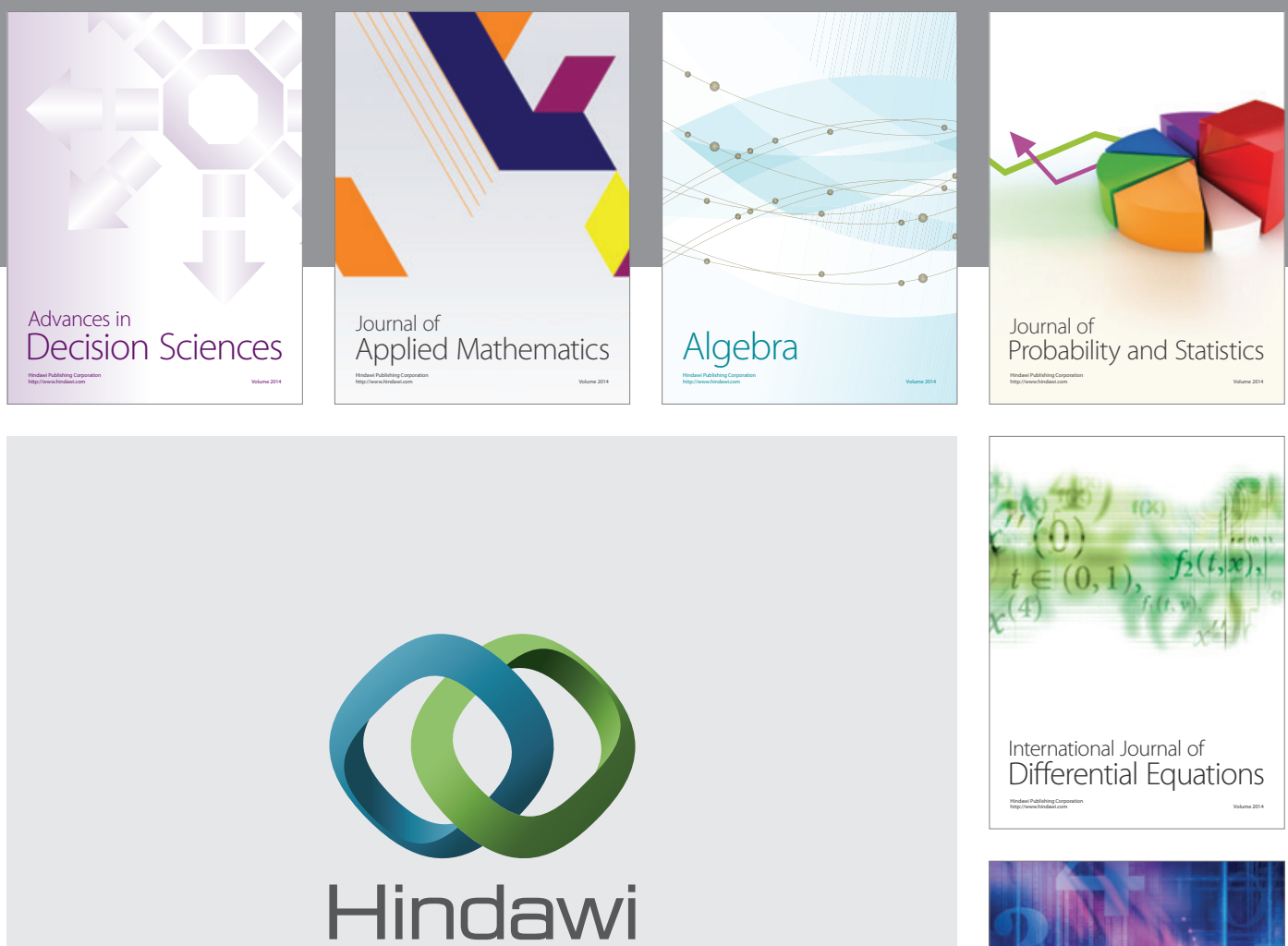

Submit your manuscripts at http://www.hindawi.com
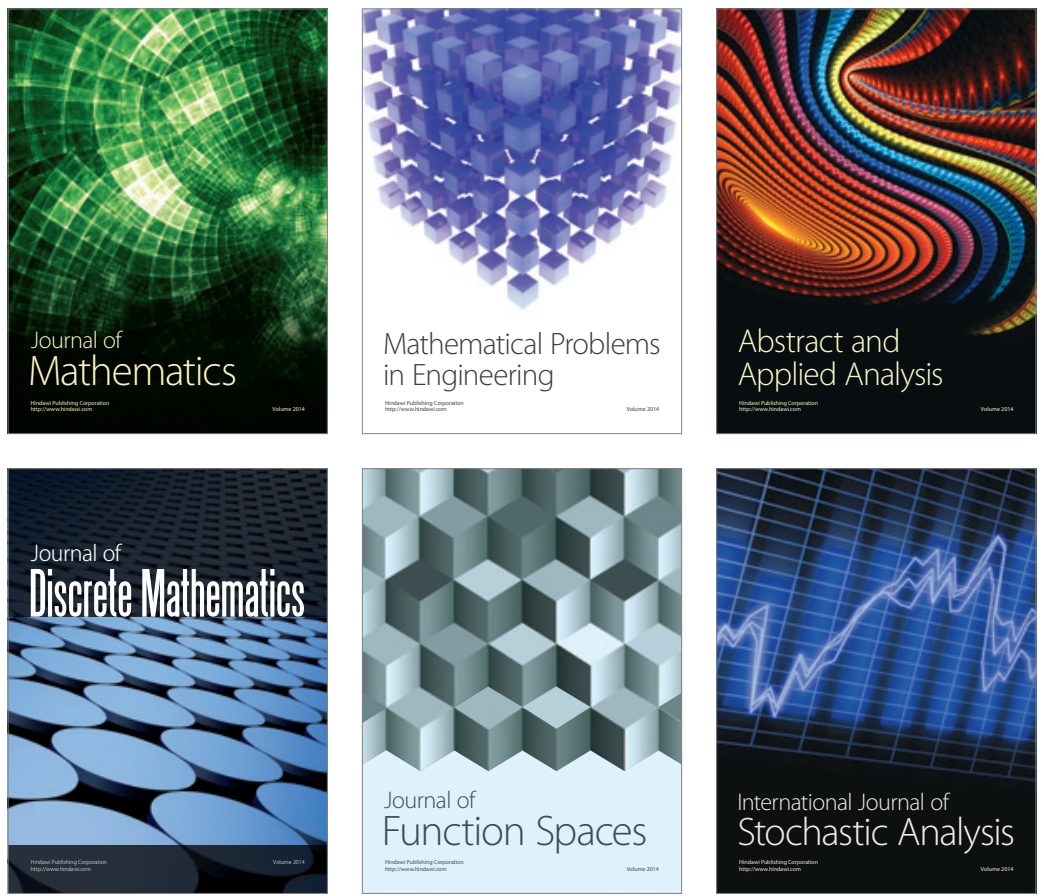

Journal of

Function Spaces

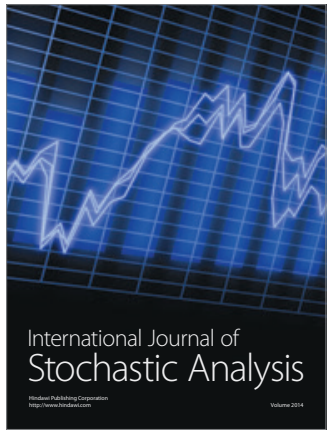

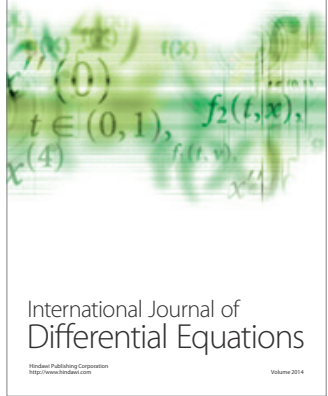
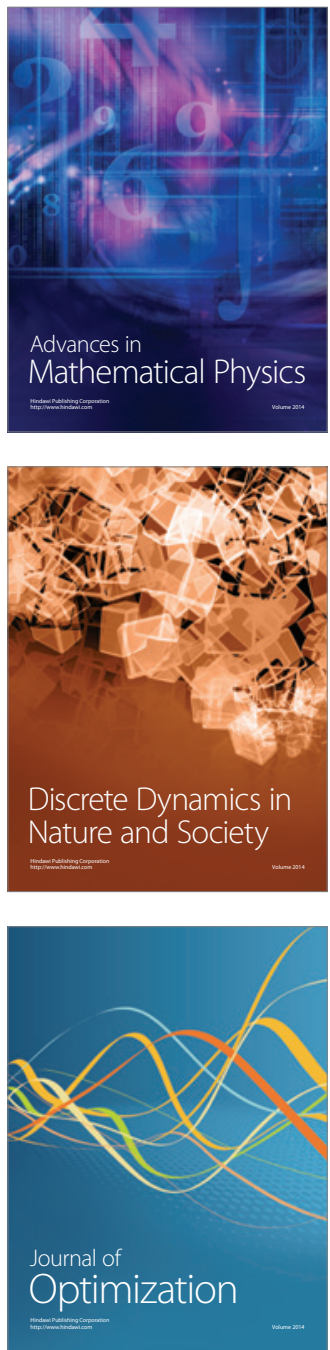